\title{
Community-acquired meningitis caused by a CG86 hypervirulent Klebsiella pneumoniae strain: first case report in the Caribbean
}

\author{
Bénédicte Melot', Sylvain Brisse ${ }^{2,3 *}$, Sébastien Breurec ${ }^{4,5,6}$, Virginie Passet ${ }^{2,3}$, Edith Malpote ${ }^{4}$, Isabelle Lamaury ${ }^{1}$, \\ Guillaume Thiery ${ }^{7,6}$ and Bruno Hoen ${ }^{1,6,8^{*}}$
}

\begin{abstract}
Background: Community-acquired bacterial meningitis due to Klebsiella pneumoniae has mainly been described in Southeast Asia and has a poor prognosis. Severe invasive infections caused by K. pneumoniae, including meningitis, are often due to hypervirulent strains (hvKP), which are characterized by capsular serotypes K1 and K2, a gene responsible for hypermucoviscosity, and the cluster for synthesis of the siderophore aerobactin.

Case presentation: A 55 year old man with a history of essential hypertension, benign prostate hyperplasia, hyperlipidemia, obstructive sleep apnea, and chronic alcoholism was admitted for meningitis due to Klebsiella pneumoniae with a wild-type susceptibility profile. Its genomic features were consistent with a capsular K2 strain belonging to clonal group 86 (CG86) displaying the large virulence of Klebsiella plasmid (pLVPK) with heavy metal resistance gene clusters, aerobactin, rmpA.
\end{abstract}

Conclusion: This is the first case of community-acquired meningitis caused by a hypervirulent strain of hvKP ever reported in the Caribbean.

Keywords: Community acquired meningitis, ST86 hypervirulent Klebsiella pneumoniae strain, Caribbean

\section{Background}

Klebsiella pneumoniae is an uncommon cause of community-acquired bacterial meningitis $[1,2]$. A new group of severe infections due to hypervirulent strains of Klebsiella pneumoniae has been described in the past few years, mainly in Southeast Asia, including liver abscesses, pneumonia, meningitis, metastatic localisations [2,3] and has a poor prognosis [4]. Severe invasive infections caused by K. pneumoniae, including meningitis, are often due to $h \nu K P$. The genomic analysis of those strains most frequently highlights capsular serotypes K1 and $\mathrm{K} 2$ and the presence of a large virulence plasmid harboring the regulator of mucoid phenotype gene $\operatorname{rmp} A$, responsible for hypermucoviscosity, and the cluster for synthesis of the siderophore aerobactin [5-7].

\footnotetext{
* Correspondence: sylvain.brisse@pasteur.fr; bruno.hoen@chu-guadeloupe.fr ${ }^{2}$ Microbial Evolutionary Genomics, Institut Pasteur, 28 rue du Dr Roux, 75724 Paris, France

${ }^{1}$ Inserm-CIC 1424 et Service de Maladies Infectieuses et Tropicales, Centre Hospitalier Universitaire de Pointe-à-Pitre, Pointe-à-Pitre, France

Full list of author information is available at the end of the article
}

A 55-year-old man was admitted to the emergency room of the University Hospital of Pointe-à-Pitre, Guadeloupe, French West Indies, on July 14th, 2014, because of consciousness deterioration and photophobia. $\mathrm{He}$ was born and had always lived in Guadeloupe. He had a history of essential hypertension, benign prostate hyperplasia, hyperlipidemia, obstructive sleep apnea, and chronic alcoholism but he neither had cirrhosis nor diabetes. On July 10th, he was given amoxicillin-clavulanate orally for an acute suppurative left otitis media without microbiological documentation. At the time of admission, he was afebrile and hemodynamically stable, but otoscopy revealed a persisting suppurative otitis media and neurological examination showed a stiff neck. White blood cell count was $11.6 \mathrm{G} / \mathrm{L}$ and serum C-reactive protein was $414 \mathrm{mg} / \mathrm{L}$. Computed tomography (CT) scan of the brain was normal. Cerebrospinal fluid (CSF) was cloudy, with the following abnormalities: glucose below limit of detection, protein $8.38 \mathrm{~g} / \mathrm{L}$, and 5,500 white blood cells $(\mathrm{WBC}) / \mathrm{mm}^{3}$, (90\% of polymorphonuclear 
(PMN) cells). Many Gram-negative bacilli were observed on direct microscopy and CSF culture grew hypermucoviscous KP with a wild-type susceptibility profile. Immediately after lumbar puncture, intravenous cefotaxime (4 g qid) was started and the patient was transferred to the intensive care unit. CT-scan of the brain showed a left mastoiditis and CT-scan of the abdomen was normal, with no liver abscess. On July 16th, brain magnetic resonance imaging (MRI) FLAIR sequences showed diffuse subcortical hypointense signal abnormalities of the supratentorial zone, which was consistent with a posterior reversible encephalopathy syndrome without edema probably related to undertreated high blood pressure and exacerbated by the sepsis. On July 18th, repeat lumbar puncture yielded cloudy CSF with improved parameters: glucose $4.8 \mathrm{mmol} / \mathrm{L}$, protein $3.0 \mathrm{~g} / \mathrm{L}, 3,093 \mathrm{WBC} /$ $\mathrm{mm}^{3}$ (87\% of PMN cells). A few Gram-negative bacilli were noted on direct microscopy but the culture remained negative. Cefotaxime (4 g qid) was continued for a total of 21 days and the patient recovered progressively. When he was discharged from hospital, he still had multiple paresis of cranial nerves (III, VI, and VII) and hemiparesia. When he was re-evaluated 3 weeks later all symptoms had disappeared and neurological examination had returned to normal.

\section{Case presentation}

To determine the genotypic characteristics of the KP isolate (SB4936), a genomic sequence was obtained using an Illumina $2 \times 300$ nt paired end protocol on a MiSeq instrument. Reads were assembled de-novo using CLCbio assembler. The genomic sequence was submitted to the European Nucleotide Archive (accession number PRJEB9692). Contigs were scanned using the BIGSdb tool (http://bigsdb.pasteur.fr) for core genome multilocus sequence typing (cgMLST) as well as KP virulence and resistance genes [8, 9]. Phylogenetic analysis of gene sequences showed that the strain belongs to KP sensu stricto [10]. Consistent with this, the genome sequence harbored the marker Kp50233, previously shown to be specific for KP [11]. Analysis of the 7-gene Multilocus sequence typing (MLST) sequences [12] showed that the isolate belongs to sequence type (ST) 86, the archetype ST of clonal group (CG) 86, previously recovered from severe community-acquired infections $[9,13]$. Furthermore, comparisons of the 694 cgMLST genes revealed only 19 allelic mismatches when compared to reference strain SA1 of ST86, which demonstrates that isolate SB4936 belongs to CG86 [9, 13]. Consistently, the genome also harbored genes kpiA and $n i k A 2$, which are typical for this clonal group [11]. It also possessed the following virulence factors: $r m p A$ and rmpA2 (regulator of mucoid phenotype, associated with the hypermucoviscous phenotype), iroBCDN (coding for salmochelin), iucABCDiutA (coding for the aerobactin siderophore cluster), $k v g \mathrm{AS}$ (a two-component system), mrkABCDFHIJ (coding for the cluster for type III fimbriae involved in adhesion and biofilm formation). Conversely the $k f u A B C$ (iron aquisition) and allABCDRS (allantoin utilization) clusters were absent from its genome. The strain also possessed gene sequences $w z i-2$ and $w z c-2$, previously shown to be specifically encountered in strain of capsular serotype K2, another important virulence factor of KP. The strain did not harbor the irp1 and irp 2 genes coding for polyketide synthase/non ribosomal peptide synthetase associated with yersinibactin siderophore synthesis. No resistance gene other than $b l a_{\mathrm{SHV}-1}$ was detected in the genome of SB4936 which was consistent with the antimicrobial susceptibility profile (resistance to only ampicillin, ticarcillin and piperacillin). The genomic features of isolate SB4936 were consistent with a hypervirulent strain with a wild-type susceptibility profile, as previously described for isolates of clonal group CG86 [8, 9, 11, 14]. The strain also harbored the heavy metal resistance gene clusters $p b r$ (lead), pco (copper), sil (silver) and ter (tellurium). The presence of these clusters as well as of $\operatorname{rmp} A$ and the aerobactin cluster demonstrates the presence in SB4936 of a plasmid similar to PLVPK, the large virulence plasmid of K. pneumoniae [5]. Overall, 249 out of 251 proteincoding genes of pLVPK were present in SB4936, among which 237 were $100 \%$ identical in nucleotide sequence.

\section{Conclusion}

We report the second case of invasive infection due to a hypervirulent community-acquired strain of KP ever identified in the Caribbean area [15] and the first case of community-acquired meningitis, possibly signaling its emergence in this part of the world.

The clinical presentation was similar to that of other published cases of meningitis caused by this strain $[3,7,16]$. Diabetes mellitus, liver cirrhosis and alcoholism have all been reported as the most significant risk factors for $K$. pneumoniae invasive severe infections $[1,2]$. As in most other cases of meningitis due to hypervirulent KP it was community-acquired $[7,16]$. This case report demonstrates that hypermucoviscous strains of $K$. pneumoniae have spread to the Caribbean. Therefore we would recommend that clinicians as well as microbiologists from this region be prepared to specifically investigate strains isolated from patients presenting with severe invasive community-acquired K. pneumoniae infections.

\section{Abbreviations}

CG: Clonal group; cgMLST: Core genome multilocus sequence typing; CSF: Cerebro-spinal fluid; CT: Computed tomography; HvKP: Hypervirulent Klebsiella pneumoniae; KP: Klebsiella pneumoniae; MRI: Magnetic resonance imaging; PLVPK: Large virulence plasmid of Klebsiella Pneumoniae; PMN: Polymorphonuclear leucocytes; ST: Sequence type; WBC: White blood cells 


\section{Acknowledgments}

We thank the technicians of the microbiology laboratory in the University Hospital of Pointe-à-Pitre/Abymes.

\section{Funding}

This work was supported by the French Government's Investissement d'Avenir program, Laboratoire d'Excellence "Integrative Biology of Emerging Infectious Diseases" (Grant no. ANR-10-LABX-62-IBEID).

\section{Availability of data and materials}

The datasets supporting the conclusions of this article are included within the article.

\section{Authors' contributions}

$\mathrm{BM}, \mathrm{BH}$, and SBre drafted the manuscript. SBri made substantial contributions to conception and design, acquisition of data, analysis and interpretation of data. VP and EM contributed to the acquisition, analysis and interpretation of data. BH, IL and GT revised the manuscript critically for important intellectual content. BH gave final approval of the version to be published. All authors read and approved the final manuscript.

\section{Competing interests}

The authors declare that they have no competing interests.

\section{Consent for publication}

Written consent to publish this case report and all accompanying images was obtained from the patient.

\section{Ethics approval and consent to participate}

Not Applicable.

\section{Methodology}

We adhered to the CARE guidelines/methodology.

\section{Author details}

${ }^{1}$ Inserm-CIC 1424 et Service de Maladies Infectieuses et Tropicales, Centre Hospitalier Universitaire de Pointe-à-Pitre, Pointe-à-Pitre, France. ${ }^{2}$ Microbia Evolutionary Genomics, Institut Pasteur, 28 rue du Dr Roux, 75724 Paris, France. ${ }^{3}$ UMR3525, CNRS, Paris, France. ${ }^{4}$ Laboratoire de Microbiologie clinique et environnementale, Centre Hospitalier Universitaire de Pointe-à-Pitre/les Abymes, Pointe-à-Pitre, France. ${ }^{5}$ Unité Environnement et Santé, Institut Pasteur de Pointe-à-Pitre, Pointe-à-Pitre Guadeloupe, France. ${ }^{6}$ Faculté de Médecine Hyacinthe Bastaraud, Université des Antilles et de la Guyane, Pointe-à-Pitre, Guadeloupe, France. 'Service de Réanimation, Centre Hospitalier Universitaire de Pointe-à-Pitre/les Abymes, Pointe-à-Pitre, France. ${ }^{8}$ Centre Hospitalier Universitaire de Pointe-à-Pitre, Service de Maladies Infectieuses et Tropicales, BP 465, 97159 Pointe-à-Pitre Cedex, France.

\section{Received: 17 March 2016 Accepted: 24 November 2016}

\section{Published online: 07 December 2016}

\section{References}

1. Tang LM, Chen ST, Hsu WC, Chen CM. Klebsiella meningitis in Taiwan: an overview. Epidemiol Infect. 1997;119:135-42.

2. Jung J, Park K-H, Park SY, Song EH, Lee EJ, Choi S-H, et al. Comparison of the clinical characteristics and outcomes of klebsiella pneumoniae and streptococcus pneumoniae meningitis. Diagn Microbiol Infect Dis. 2015;82:87-91.

3. Shon AS, Bajwa RPS, Russo TA. Hypervirulent (hypermucoviscous) klebsiella pneumoniae: a new and dangerous breed. Virulence. 2013;4:107-18.

4. Ko W-C, Paterson DL, Sagnimeni AJ, Hansen DS, Von Gottberg A, Mohapatra S, et al. Community-acquired klebsiella pneumoniae bacteremia: global differences in clinical patterns. Emerg Infect Dis. 2002;8:160-6.

5. Chen Y-T, Chang H-Y, Lai Y-C, Pan C-C, Tsai S-F, Peng H-L. Sequencing and analysis of the large virulence plasmid pLVPK of klebsiella pneumoniae CG43. Gene. 2004;337:189-98.

6. Tang H-L, Chiang M-K, Liou W-J, Chen Y-T, Peng H-L, Chiou C-S, et al. Correlation between klebsiella pneumoniae carrying pLVPK-derived loci and abscess formation. Eur J Clin Microbiol Infect Dis. 2010;29:689-98.
7. Yu VL, Hansen DS, Ko WC, Sagnimeni A, Klugman KP, von Gottberg A, et al. Virulence characteristics of klebsiella and clinical manifestations of K. Pneumoniae bloodstream infections. Emerg Infect Dis. 2007;13:986-93.

8. Bialek-Davenet S, Nicolas-Chanoine M-H, Decré D, Brisse S. Microbiological and clinical characteristics of bacteraemia caused by the hypermucoviscosity phenotype of klebsiella pneumoniae in Korea. Epidemiol Infect. 2013;141:188.

9. Bialek-Davenet S, Criscuolo A, Ailloud F, Passet V, Jones L, Delannoy-Vieillard A-S, et al. Genomic definition of hypervirulent and multidrug-resistant Klebsiella pneumoniae clonal groups. Emerg Infect Dis. 2014;20:1812-20.

10. Brisse $S$, Passet $V$, Grimont PAD. Description of klebsiella quasipneumoniae sp. nov., isolated from human infections, with two subspecies, klebsiella quasipneumoniae subsp. Quasipneumoniae subsp. nov. And klebsiella quasipneumoniae subsp. Similipneumoniae subsp. nov., and demonstration That klebsiella singaporensis is a junior heterotypic synonym of klebsiella variicola. Int J Syst Evol Microbiol. 2014;64:3146-52.

11. Bialek-Davenet S, Criscuolo A, Ailloud F, Passet V, Nicolas-Chanoine M-H, Decré $D$, et al. Development of a multiplex PCR assay for identification of klebsiella pneumoniae hypervirulent clones of capsular serotype K2. J Med Microbiol. 2014;63:1608-14.

12. Diancourt L, Passet V, Verhoef J, Grimont PAD, Brisse S. Multilocus sequence typing of Klebsiella pneumoniae nosocomial isolates. J Clin Microbiol. 2005:43:4178-82.

13. Decré $D$, Verdet C, Emirian A, Le Gourrierec T, Petit J-C, Offenstadt G, et al. Emerging severe and fatal infections due to klebsiella pneumoniae in two University Hospitals in France. J Clin Microbiol. 2011;49:3012-4.

14. Lin J-C, Koh TH, Lee N, Fung C-P, Chang F-Y, Tsai Y-K, et al. Genotypes and virulence in serotype K2 klebsiella pneumoniae from liver abscess and noninfectious carriers in Hong Kong, Singapore and Taiwan. Gut Pathog. 2014;6:21.

15. Doud MS, Grimes-Zeppegno R, Molina E, Miller N, Balachandar D, Schneper L, et al. A k2A-positive Klebsiella pneumoniae causes liver and brain abscess in a Saint Kitt's man. Int J Med Sci. 2009;6:301-4.

16. Siu LK, Yeh K-M, Lin J-C, Fung C-P, Chang F-Y. Klebsiella pneumoniae liver abscess: a new invasive syndrome. Lancet Infect Dis. 2012;12:881-7.

\section{Submit your next manuscript to BioMed Central and we will help you at every step:}

- We accept pre-submission inquiries

- Our selector tool helps you to find the most relevant journal

- We provide round the clock customer support

- Convenient online submission

- Thorough peer review

- Inclusion in PubMed and all major indexing services

- Maximum visibility for your research

Submit your manuscript at www.biomedcentral.com/submit
) Biomed Central 